\title{
PANDA EMC Trigger and Data Acquisition Algorithms Development
}

\author{
Qiang Wang ${ }^{* a, b}$, Dapeng $\operatorname{Jin}^{b}$, Andreas Kopp ${ }^{a}$, Wolfgang Kühn ${ }^{a}$, Jens Sören Lang ${ }^{a}$, \\ Yutie Liang ${ }^{a}$, Ming Liu ${ }^{a}$, Zhen-an Liu ${ }^{b}$, David Münchow $^{a}$, Björn Spruck ${ }^{a}$ and Hao Xu ${ }^{b}$ \\ ${ }^{a}$ Physics Institute II, Giessen University \\ Heinrich-Buff-Ring 16, D-35392, Giessen, Germany \\ ${ }^{b}$ Experimental Physics Center, Institute of High Energy Physics, Chinese Academy of Sciences \\ 19B Yuquan Road, Shijingshan District, 100049, Beijing, China \\ E-mail: Qiang.Wang@physik.uni-giessen.de, jindp@ihep.ac.cn, \\ Andreas.Koppdexp2.physik.uni-giessen.de, \\ Wolfgang.Kuehn@exp2.physik.uni-giessen.de \\ Soeren.Langedexp2.physik.uni-giessen.de, \\ Yutie.Liangdexp2.physik.uni-giessen.de, \\ Ming.Liudexp2.physik.uni-giessen.de, liuzadihep.ac.cn, \\ David.Muenchowdexp2.physik.uni-giessen.de \\ Bjoern.Spruckdexp2.physik.uni-giessen.de xuhaodihep.ac.cn
}

The PANDA detector is a general purposed hadron spectrum planed to operate at the FAIR facility in Darmstadt, Germany. The PANDA electromagnetic calorimeter(EMC) employs 11360 PWOII crystals for barrel module, 3600 for forward module and 592 for backward module providing $4 \pi$ spatial coverage, good granularity and high energy resolution. A novel self-trigger data push data architecture for the PANDA data acquisition system requiring the data from EMC readout electronics to be processed on the fly to reconstruct electromagnetic shower. Features extracted from the electromagnetic shower combine with information from other detectors such as tracking and Cherenkov detectors in order to discriminate between photons, electron and hadrons. The PANDA EMC detector performance, proposed readout electronics and offline reconstruction algorithms are studied and an adaptive EMC DAQ schematic is proposed based on an FPGA based Compute Node which provides high bandwidth flexible connections between processing modules, up to 10GByte DDR2 per board for data buffering and five large capacity V4FX60 FPGAs for sophisticated algorithms applications. The algorithms partition strategy is proposed considering the readout electronics layout and the Compute Node based processing architecture. The function verification and performance evaluation methods for the algorithms are also studied.

XLVIII International Winter Meeting on Nuclear Physics, BORMIO2010

January 25-29, 2010

Bormio, Italy

\footnotetext{
${ }^{*}$ Speaker.
} 


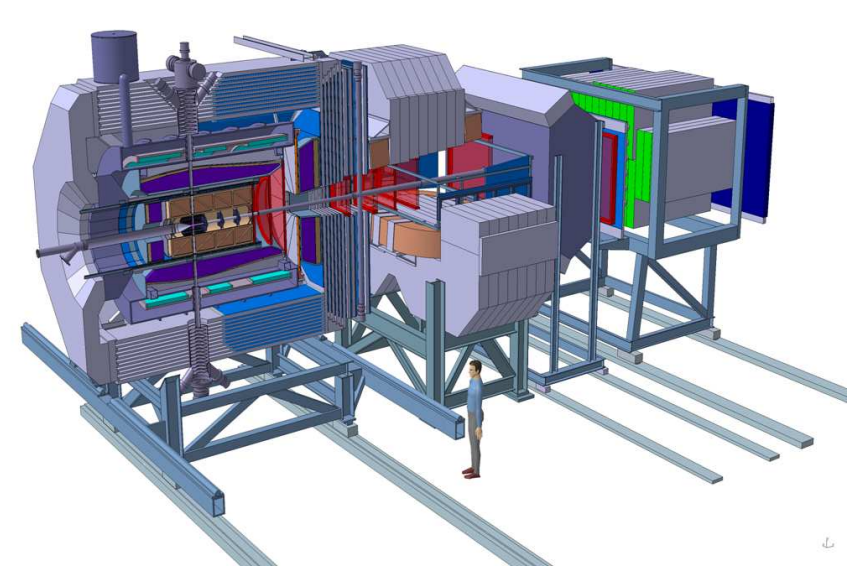

Figure 1: The PANDA detector is composed of a target spectrometer, surrounding the interaction region, and a forward spectrometer to detect emitted particles at forword angle. The target spectrometer consists of a Micro Virtex detector, Central Tracker, Barrel TOF, DRIC detector, EMC detector, solenoid and Muon detector from inside to out side.

\section{Introduction}

The PANDA experiment has a comprehensive physics program which includes charmonium spectroscopy, searching for QCD exotica, hypernuclear physics, measurement of timelike form factors of proton and so on[1]. This rich and diverse physics programme requires high luminosity experiments with interaction rates of up to $20 \mathrm{MHz}$. Raw data rates of about $200 \mathrm{GByte} / \mathrm{s}$ need to be processed in real time to select events of interest in a potentially very large background.

A conceptual design of PANDA detector is shown in fig.1. The EMC detector are set outside the track detector and inside the magnet coil. A optimal PWOII scintillator is planed to be implemented on the PANDA experiment which features fast time response, high density, improved light yield and low material cost. The EMC detector provides very good energy resolution, the PROTO60 test experiment[2] shows a energy resolution of better than $2.4 \%$ at $1 \mathrm{GeV}$. Good spatial resolution is very important for $\pi^{0}$ reconstruction. The smallest possible open angular of the final state photons from $\pi^{0}$ decay is about 2 degree for barrel part[3] which is just one crystal width. Overlapped bumps in one cluster can be processed to construct the original showers if obvious peaks exist. The major role for EMC trigger and data acquisition system is to reconstruct deposited energy and position information for photons, electrons and hadrons from the electromagnetic showers. Cluster shape information can also be used to provide some particle identification information for hadrons. The integrated single crystal hit rate ranges from $5 \mathrm{kHz}$ to $60 \mathrm{kHz}$ for the barrel part and $100 \mathrm{kHz}$ to $500 \mathrm{kHz}$ for the forward endcup[3], fast processing speed should be a main feature of the high level trigger algorithms. Considering possible large latency for complicated algorithms and time needed to collect information for high level correlations, huge data buffering capability should also be satisfied. 


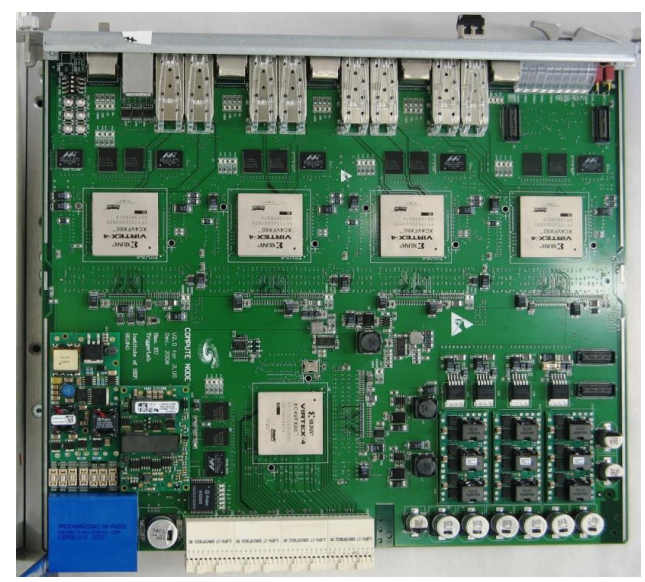

Figure 2: Compute node version2 hardware top view.

\section{PANDA EMC trigger and DAQ schematic using an FPGA based compute node}

\subsection{Features of compute node}

To fulfil the special requirements as mentioned above, an FPGA based compute node $(\mathrm{CN})$ is developed as the basic hardware module to build a huge trigger and DAQ system. Fig2. shows a top view of the PCB layout of the compute node version2. The compute node follows a new ATCA standard, in which high speed serial links are available for backplane P2P connections. 13 RocketIO based high speed serial links are used for interconnection between each two modules in the same shelf so that a full mesh P2P network can be formed. Five Virtex4FX60 FPGAs provide huge processing power and each are combined with a 2GByte DDR2 memory for large data buffering. On the front panel, 5 Gigabit Ethernet ports and 8 optical links are used for massive data transmission. To easier large system design, a system-on-programmable-chip design topology is implemented[4]. A general-purpose embedded system is built using the embedded PowerPC405 hardcore and some open source IP cores. An open source Linux system is ported to the platform for web server, slow control, tcp/ip package processing and other not time critical tasks. The dedicated trigger algorithms such as cluster finder for EMC, helix tracking algorithm are designed as custom processing units with FPGA fabric resources. These customized processing units are combined with other general purposed modules to build a large system on chip.

\subsection{PANDA EMC TDAQ schematic}

Based on the compute node and read out electronics, an EMC TDAQ schematic is proposed as shown in Fig.3. Data from read out modules are forwarded to compute node via optical links. At the first stage, only sub-detectors information is processed. For EMC detector, cluster finding, cluster shape parameters extraction and pattern recognition should be done. All sub-detector information are combined together for high level correlation. Some physics parameters can be calculated at this stage and particle identification can also be done. Finally data will be sent to the PC farms after event building. 


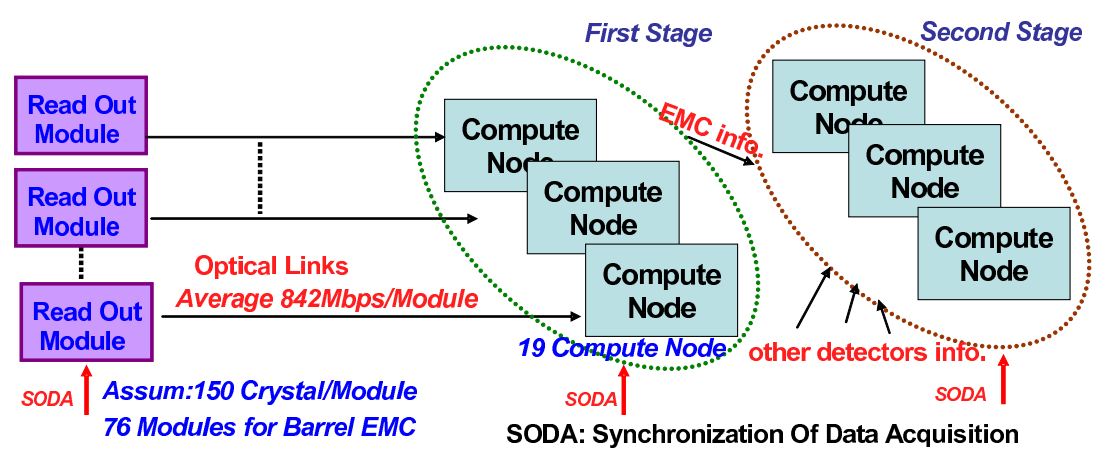

\begin{tabular}{|l|l|l|l|}
\hline \begin{tabular}{|l|l|} 
1. Signal Feature extraction \\
(Time, Amplitude)
\end{tabular} & $\begin{array}{l}\text { 1. Clustering } \\
\text { 2. Cluster Properties } \\
\text { extraction } \\
\text { 2. Data Zero suppression }\end{array}$ & $\begin{array}{l}\text { 1. Correlation } \\
\text { 2. Physical parameters } \\
\text { calculation } \\
\text { 3. Eattern recognition }\end{array}$ \\
\hline
\end{tabular}

Figure 3: EMC data aquisition schematic based the compute node and tasks for each stage to fulfil.
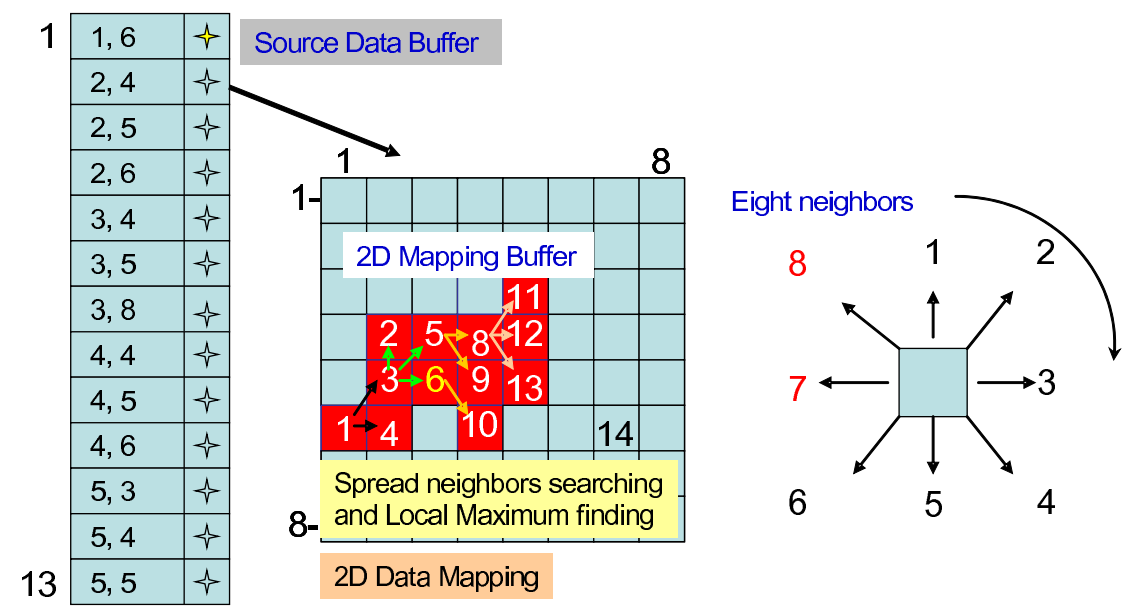

Figure 4: A snapshot of spread neighbors searching algorithm.

\section{Algorithms development}

In offline cluster finding algorithm, one hit needs to compare with all the other hits in same event to find clusters. It is every time consuming when hit multiplicity goes up to 100 or event more for the PANDA experiment. Additionally, complex data structures and float point calculations are not fit for FPGA implementation. Comparing with the offline algorithm, in online cluster finder, hits belong to the same cluster firstly are mapped to an tow dimensional buffer based on the two coordinator index of crystals. A hit filtering algorithm is needed if there are too many isolated hits appear when the single crystal energy threshold is very low or noise level is very serious. As show in Fig.4, a spread neighbors searching process starts at one hit and all its possible neighbors in eight directions are checked. Found neighbors are buffered as the seeds for following neighbors 
searching. After all possible neighbors are checked, all hits belong to one cluster are found and buffered for next stage processing. During the cluster searching process, a local maximum finding algorithm is used to check if found cluster is overlapped one or not. A restrict selection criteria is used to avoid fake local maximus found. Position and energy information is needed for correlation with track detector. Bumps splitting is necessary before position and energy calculation. For cluster position calculation, two methods are considered. One is a common linear weight gravity position calculation and the other is combined logarithmic and linear weight position calculation[3]. The first one is much easier to be implemented in FPGA based computation platform but less precise comparing with the second one. Future studies are need to decide which one is more suitable. After all these have been done, cluster information like energy, position, size, and multiplicity can be provide for next stage processing.

Cluster shape parameters can be used to help hadrons identification. In offline algorithms, cluster shape parameters like polar angle, $\mathrm{E}_{1} / \mathrm{E}_{9}, \mathrm{E}_{9} / \mathrm{E}_{25}$, lateral moment, zernike moments are extracted. These shape parameters can be used as input of a neural network to identify particles between $\pi, \mathrm{K}, \mathrm{P}$ and $\mu[3]$. Some shape parameters calculation are complicated and deep studies are needed to see if they are fit for FPGA implementation in our compute node or left for CPU based calculation in PC farms.

\section{Summary}

In the first part, the main features of PANDA experiment and its detector system are introduced and its specific requirements on the trigger and DAQ system are analyzed. Then an adaptive EMC trigger and DAQ system schematic is proposed based on a universal hardware platform. In view of the limitations on offline reconstruct algorithms, a fast cluster finder algorithm is proposed and the corresponding processing flow is described. The algorithms written in $\mathrm{C}$ language are under progress and some modules have been finished and tested with simulation data.

\section{Acknowledgments}

This work was supported in parts by BMBF under contract No. 06GI9107I, China Project"973" under contract No.2008CB817702, HIC for FAIR, WTZ-CN(06/09) and DAAD-CAS Scholarship.

\section{References}

[1] $\bar{P} A N D A$ Collaboration, Technical Progress Report for PANDA-Strong Interaction Studies with Antiprotons, 2005.

[2] P.A.Semenov, et al., PANDA electromagnetic calorimeters, Nucl. Instr. and Meth. A 598 (2009) 224-228.

[3] P̄ANDA Collaboration, Technical Design Report for PANDA Electromagnetic Calorimeter(EMC)-Strong Interaction Studies with Antiprotons, 2008.

[4] Qiang Wang, et al., Hardware/Software Co-design of an ATCA-based Computation Platform for Data Acquisition and Triggering, Real Time Conference, 2009, RT'09. 16th IEEE-NPSS 485-489. 